\title{
Über die Frage, ob neben dem Pepsin ein anderes Enzym, Chymosin, anzunehmen ist.
}

Von

\section{A. Pekelharing.}

Die Labwirkung auf Milch scheint so gan' und gar verschieden von der Pepsinwirkung auf Eiweissstoffe, dass man es anfänglich als selbstverständlich betrachtet hat, dass diese beiden Wirkungen zwei unter sich verschiedenen Enzymen zugeschrieben werden müssten. Im einen Fall gerinnt die Milch hei neutraler oder sehr schwach saurer Reaktion, während im anderen eine sehr deutlich saure Reaktion notwendig ist und das Eiweiss gelöst oder, wenn es sich schon in Lösung befindet, solcherart veländert wird, dass es, je länger die Wirkung dauert, allmählich schwieriger zu fällen wird.

Die weitere Forsehung aber lehrte, dass der Unterschied nicht so gross ist, wie es anfangs schien.

Schon vor 20 Jahren habe ich mitgeteilt, dass allerhand Pepsinpräparate imstande sind, bei neutraler Reaktion typische Milchgerinnung hervorzurufen, auch dann, wenn das Pepsin aus einem Schweinsmagenschleimbautinfus bereitet worden war, welches mit $0,5 \% \mathrm{HCl} 5$ Tage lang bei Körpertemperatur digeriert war und dabei nahezu ganz chymosinfrei geworden zu sein schien ${ }^{1}$ ).

Einige Jahre später fanden $\mathrm{Paw}$ low und $\mathrm{Par}$ astscbuk ${ }^{2}$ ), dass im Magensaft des Hundes das Vermögen, Milch festzulegen, immer mit dem proteolytischen Vermögen gleichen Schritt hält. Ausserdem hatte es sich inzwischen herausgestellt, dass proteolytische Enzyme überhaupt, von pflanzlichem oder von tierischem Ursprung, auch bei neutraler oder sehr schwach saurer Reaktion, Milchgerinnung hervorrufen können. Pawlow und Parastschuk untersuchten auch den vom Pylorusteil der Magenschleimhaut ausgeschiedenen Saft, den

1) Zeitschr. f. physiol. Chemie Bd. 22 S. 244.

2) Zeitschr. f. physiol. Chemie Bd. 42 s. 415. 
Pankreassaft und das Ausscheidungsprodukt der Brunner'schen Drüsen beim Hund und konnten nachweisen, dass in allen diesen Säften die labende Kraft der proteolytischen nahezu proportional war. Auch fanden sie, dass die aktivierende Wirkung der Enterokinase auf Pankreassaft für beide Enzymwirkungen dieselbe war. Diese Befunde führten Pawlow und Parastschuk zu dem Schluss, dass Chymosin dasselbe Enzym ist als Pepsin, dass aber die Wirkung von den Umständen abhängig ist. Sie waren geneigt anzunehmen, dass die Gerinnung des Kaseins und die Verdauung von Eiweiss unter dem Einfluss desselben Enzyms als ein Beispiel zu betrachten sein würde von entgegengesetzter Enzymwirkung, welche nicht nur aus theoretischen Gründen postuliert, sondern auch in bezug anf Kohlehydrat- und fettspaltende Enzyme tatsächlich nachgewiesen worden war.

Fin Finwurf, gegen diese Auffassung angeführt, dass es im Handel Lahpräparate gibt, welche stark labend wirken und Fiweiss nicht verdauen, stelite sich als nicht zutreffend heraus. ['a w] ow kounte nachweisen, dass in solchen Iräjaraten die verdauende Kraft nicht fehlt, sondern nur maskiert ist infolge der Anwesenheif von Beimiscliungen, welche mittels Verdünnung mit Salzsäure oder, hesser noch, mittels Dialyse gegren Salzsäure fortgeschafft werden können.

Später haben mehrere Forscher sich mit der Frage beschäftigt: Ïbereinstimmung ist aber noch nieht erreicht. Iusibsondere $\mathrm{Ham}$ marsten hat an der Auffassung, dass Pepsin und Chymosin zwei verschiedene Enzymo sind, festgelialten und dieselbe auf Grund zahlreicher, mit grösster Sorøfalt angestellten Versuche kriiftig verteidigt.

Es ist nicht meine Absicht, diesen Gegenstand ausführlich und in Besonderheiten $z u$ besprechen. Ich wünsthe nur einige Beobachtungen mitzuteilen, welche auf einen Punkt Beziehung haben, der meiner Ansicht in dieser Streitfrage wichtig ist.

Weil nicht nur Infuse aus der Magenschleinhaut des Kalbes, sondern auch solche aus der des Schweines und des Hundes, auch der reibe Magensaft des Hundes Milchgerinnung hervorzurufen imstande sind, hat man anfänglich natürlicherweise angenommen, dass in allen diesen Fällen das Tnfus oder der Magensaft neben Pepsin auch Chymosin enthielt. Es wuiden aher Unterschietle gefunden, welche Bang zu dem Schluss gebracht haben, dass in Extrakteu der Magenschleimhaut des Schweines und in Handelspräparaten von 
Pepsin das labende Enzym vom Chymosin des Kalbes untersehieden werden muss. Fr nannte es deshalb Parachymosin r).

Gew in hat die Richtigkeit dieser Unterscheidung nicht bestätigen können ${ }^{2}$ ). Er fand, dass der Unterschied zwischen "Chymosin" und "Parachymosin" abnimmt, je nachdem das Chymosin besser gereinigt worden ist. Bang hatte gefunden, dass Chymosin mittels Erhitzen der sehr schwach-sauren Lösung während 10 Minuten auf $70^{\circ} \mathrm{C}$. unwirksam gemacht wird, indem Parachymosin bei dieser Behandlung nur wenig an Kraft verliert, dass dagegen Parachymosin durch kurz danernde Behandlung mit Alkali viel stärker als Chymosin angegriffen wird.

Es sei mir erlaubt, einen von Gewin's hierauf bezüglichen Versuchen anzuführen.

Ein mittels $0,4 \% \mathrm{HCl}$ hergestelltes Kalbsmageninfus wurde 24 Stunden gegen strömendes Wasser dialysiert und auf der Zentrifuge von der dabei entstandenen Fällung befreit. Ein Teil der abgegossenen Flüssigkeit wurde durch Zusatz von $\mathrm{NaOH}$ äusserst schwach-sauer gemacht, ein anderer Teil wurde alkalisch gemacht bis zu einem Gehalt von $0,01 \% \mathrm{NaOH}$ und nach einer halben Stunde mit so viel $\mathrm{HCl}$ versetzt, dass die Reaktion wieder genau so schwach-sauer war als zuvor. Ein Teil der nicht alkalisch gemachten Flüssigkeit wurde 10 Minuten auf $70^{\circ} \mathrm{C}$. erhitzt und dann sogleich abgekühlt. Bei den Gerinnungsversuchen wurde - in Übereinstimmung mit Bang - folgendes gefunden :

\section{Unverändert}

Erhitzt

Mit Alkali behandelt

Geronnen in: 20 Sekunden gerinnt nicht

35 Sekunden

Anders war es aber, wenn nicht das Infus, sondern das daraus in der von mir angegebenen Weise so rein wie möglich bereitete Enzym untersucht wurde. Dann wurde in zwei Versuchen gefunden:

$$
\text { Unverändert }
$$

I. Geronnen in:

II.

$3^{1 / 2}$ Minuten

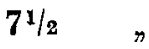

Erhitzt Mit Alkali behandelt $7^{1 / 2}$ Minuten gerinnt nicht 11

Hier hatte also das Chymosin nach der Reinigung, in bezug auf den Einfluss von Erhitzen und von Alkali, die Eigensehaften des Parachymosins erhalten.

1) Pflüger's Arch. Bd. 79 S. 425.

2) Zeitschr. f. physiol. Chemie Bd. 54 S. 53. 
Gewin kam also zu dem Schluss, dass das Kalbsmageninfus Stoffe enthält, welche das Enzym gegen die schädliche Wirkung vou Alkali $z$ beschützen imstande sind, es aber empfindlicher machen für Hitze. Fr fand weiter, dass aus dem Infus aus Kalbsmagenschleimhaut das Enzym sich viel schwieriger reinigen lässt als aus demjenigen der Schweinsschleimhaut, und schreibt es diesem Umstande $\mathrm{zu}$, dass es ihm nicht immer zu bewirken gelang, dass das Chymosin des Kalbes die Eigenschaften des Parachymosins annahm.

Auch aus einem Handelspräparat, Van Hasselt's Lab, konnte er ein Fnzym herstellen, welches, je besser es gereingt war, desto mehr, in bezug auf Alkaliwirkung und anf Frhitzung, sich dem I'arachymosin näherte.

Aus seinen in mehreren Richtungen fortgesetzten Versuchen folgerte Gewin, dass der Bestandteil von Magenschleimhautextrakten und von Magensaft, welcher Milch zur Gerinnung bringen kann, dasselbe Enzym ist: Pepsin, welches unter anderen Verhältnissen proteolytische Wirkung zeigt, und dass das Enzym im Kalbsmageninfus infolge von Verunreinigungen, welche es bis jetzt nicht völlig zu entfernen gelang, nur scheinbar von Pepsin anderer Herkunft versehieden ist.

V a n D a m, der sich eingehend mit diesem Gegenstand heschäftigt hat, hat sich der Gew in'schen Auffassung angeschlossen '). Andere Forscher aber halten den Beweis, dass im Kalbsmageninfus Stoffe enthalten sind, welche die Eigenschaften und die Wirkung zu ändern vermögen, für keineswegs geliefert.

Rakoczy gibt za, dass Pepsin ebenso wie Chymosin imstande ist, Milch zur Gerinnung zu bringen, und dass bei vielen Tieren „Hie milehkoagulierende Wirkung (Parachymosin) dem Pepsin angehört und unzertrennlich mit der eiweissverdauenden Wirkung verbunden ist ${ }^{2}$ ). Die Magenschleimhaut des Kalbes aber liefert, seiner Meinung nach, neben Pepsin auch Chymosin, ein Enzym, welches sicher nicht als verunreinigtes Pepsin betrachtet werden darf.

H a m marsten lässt die Frage, ob das Pepsin selbst auch die Milch koagulieren kann, offen ${ }^{3}$ ). In bezug hierauf erlaube ich mir zu bemerken, dass, wie ich oben schon in Erinnerung gebracht

1) Zeitschr. f. physiol. (hemie Bd. 64 S. 316 .

2) Zeitschr. f. physiol. Chemie Bd. 84 S. 334 .

3) Zeitschr. fo physiol. Chemie Bd. 94 S. $32 \%$. 
habe, möglichst gereinigtes Pepsin vom Hund oder vom Schwein, in neutraler Lösung mit Milch gemischt, Gerinnung bervorruft. Im Laufe der Jahre habe ich mich öfters von der Richtigkeit dieser Beobachtung aufs neue überzengt. Ebenso wie das Lab aus dem Kalbsmagen wirkt das Pepsin bei neutraler Reaktion in gleicher Konzentration nicht so schnell wie bei saurer, sonst wird aber das Kasein in ganz derselben Weise verändert. Es gibt, wie ich glaube, keinen einzigen Grund für die Annahme, dass die Wirkung irgendeinem anderen trotz der Reinigung dem Pepsin anhaftendem Enzym zugeschrieben werden muss. Alles, was von Parachymosin gesagt ist, hat, wie auch Rakoc\%y amimmt, für Pepsin Geltung. Ich führe hier ein Beispiel an:

$10,5 \mathrm{mg}$ aus dem Magen des Hundes bereitetes 1'epsin wurdo fein zerrieben, in 10,5 ccm 0,9\% iger Kochsalzlösung aufgenommen. Das Pepsin lost sich dabei nur trilweise, die Mischung war aber wohl gleichmässig genug, dass beim Abmessen mit der Pipette erhebliche Fehler ausgeschlossen werden konnten. $1 \mathrm{ccm}$ - also $1 \mathrm{mg}$ Pepsin, - mit $10 \mathrm{ccm}$ Milch bei $32^{\circ} \mathrm{C}$. vernischt, verursachte in 21 Minuten vollständige Gerinnung, indem $1 \mathrm{ccm}$ mit $9 \mathrm{ccm} 0,1 \%$ igem $\mathrm{HCl}$ und $50 \mathrm{mg}$ Karminfibrin in 15 Minuten so viel Fibrin gelöst hatte, dass beim Vergleich mit einer willkürlichen Verdaunngslösung von Karminfibrin, der Grützner'sche Kolorimeter Teilstrich 4 anwies.

Die noch übrige Flüssigkeit - 8,5 $\mathrm{ccm}$ - wurde jetzt mit sechs Tropfen $\frac{\mathrm{n}}{10}$-Natronlauge alkalisch gemacht und 15 Minuten später mit $\frac{\mathrm{n}}{10}$-Salzsäure genau neutralisiert, mit Lackmus als Indikator. Hiervon wurden $2 \mathrm{ccm}$ bei $32^{\circ} \mathrm{C}$, mit $10 \mathrm{ccm}$ Milch vermischt und $2 \mathrm{ccm}$ mit $50 \mathrm{mg}$ Karminfibrin in $8 \mathrm{ccm} 0,1 \%$ igem $\mathrm{HCl} 15$ Minuten digeriert und nach der Filtration mit derselben Vergleichslösung wie zuvor im Kolorimeter beobachtet. Die Milch zeigte nach 21/4 Stunden anfangende Gerinnung und war erst nach 4 Stunden vollständig geronnen, indem der Kolorimeter Teilstrich 1,3 anwies. Die verdauende Kraft hatte also, infolge der Alkaliwirkung bis auf $1 / 6$ abgenommen, die labende Kraft, welche nicht genau zu bestimmen war, nur wenig mehr, in Übereinstimmung mit der wohlbekannten Frfahrung, dass bei neutraler Reaktion die Gerinnungszeit bei Verdünnung der Enzymlösung mehr verlängert wird, als der Konzentrationsabnahme entspricht, und zwar wie $\mathrm{V}$ an $\mathrm{Dam}$ es wahrscheinlich gemacht hat, infolge der schädlichen Einwirkung der Hydroxylionen der Milch auf das Enzym; Zusatz einer sehr geringen Säuremenge, bevor das Enzym mit der Milch gemischt wird, verbessert die Wirkung ${ }^{1}$ ).

1) Zeitschr. f. physiol. Chemie Bd. 64 S. $316^{\circ}$ 
Aus diesem Beispiel geht hervor, dass die beiden Wirkungen des Pepsins durch Alkali in gleichem Maasse geschädigt werden. Bei längerer Einwirkung des Alkalis würden die verdauende und die labende Kraft ganz verloren gegangen sein.

Dass die Magenschleimhaut des Hundes und des Schweines kein "typisches Chymosin" liefert, gibt Ha mmarsten selbst zu. Soweit mir bekannt ist, hat man für die Annahme, dass das in der von mir angegebenen Weise gereinigte Pepsin irgendein anderes chymosinähnliches Enzym enthalten würde, niemals einen Grund angeführt. Bedenkt man dabei, dass auch die Bildung von Parakasein aus Kasein auf Hydrolyse beruht, wie es insbesondere von Frl. Van Herwerden betont worden ist ${ }^{1}$ ), so ist, wie es mir scheint, die Hypothese, dass es nicht das Pepsin, sondern ein anderes Enzym im Mageninfus des Schweines oder im Magensaft des Hundes ist, welches die Gerinnung der Mileb verursacht, nicht wohl zu verteidigen.

Die Gründe, auf welche die Existenz eines von Pepsin verschiedenen Chymosin angenommen wird, beruhen dann auch ausschliesslich auf an Infusen oder daraus hergestellten Präparaten von Kalhsmagen erhaltenen Befunde. In mehrfacher Weise ist es möglich, solche Infuse derartig $z u$ beeinflussen, dass entweder die eiweissverdauende oder die labende Wirkung so gut wie ganz versehwindet, während die andere erhalten bleibt.

Pawlow, Gewin, Van Dam und andere halten dafür, dass das Verschwinden der einen Wirkung, während die andere sich noch nachweisen lässt, der Anwesenheit von Verunreinigungen zugeschrieben werden muss. In gewissen Fällen ist die Richtigkeit dieser Auffassung nachzuweisen. Die im Handel vorkommenden Labpräparate verdauen Eiweiss kaum oder gar nicht. Werden sie aber gegen verdünnte Salzsäure dialysiert, so werden ihnen Beimischungen entzogen, ' und es kommt die verdauende Wirkung ans Licht. Hier ist also der Grund für die Annahme zweierlei Enzyme hinfällig. Das Handelslab kann ebensogut Eiweiss verdauen als Milch laben, wenn es nur von Stoffen, welche der Verdauung entgegenwirken, von Salzen zum Beispiel, welche die Schwellung des Eiweisses unter dem Einfluss der Säure hindern ${ }^{2}$ ), befreit wird.

In anderen Fällen aber hat man die Richtigkeit der Annahme, die Aufhebung einer der zwei Wirkungen würde dem Einfluss von

1) Zeitschr. f. physiol. Chemie Bd. 52 S. 184.

2) Vgl. Ringer, Zeitschr, f. physiol. Chemie Bd. 95 S. 227. 
Beimischungen zuzuschreiben sein, nicht nachweisen können. In seiner letzten Mitteilung ${ }^{1}$ ) hat Hammarsten noch sehr wichtige Beobachtungen veröffentlicht über sehr wenig feste Stoffe enthaltende Enzymlösungen aus der Magenschleimhaut des Kalbes, welche durch Behandlung mit Alkali die eiweissverdauende Kraft beinahe ganz verloren hatten, wäbrend die labende Kraft kaum verringert wurde Beobachtungen, welche $\mathrm{Hammarsten}$ völlig unerklärlich erachtet, wenn man annimmt, dass die Lösung nur ein einziges Enzym enthält.

Dennoch können, wie es mir scheint, diese und andere von Hammarsten angeführten Befunde auch noch nicht als für die Dualität von Pepsin und Chymosin beweisend betrachtet werden, weil sie sich auf Infuse aus der Magenschleimhaut des Kalbes beziehen, aus welchen das Enzym, Pepsin wenigstens, bei weitem nicht so rein zu erhalten ist, wie das beim Pepsin des Hundes und des Schweines der Fall ist. Dass es Stoffe gibt, welche auch in sehr geringer Menge einen deutlichen Einfluss auf die Wirkung eines Enzyms auszuüben imstande sind, ist ja nicht zu bezweifeln. In bezug auf P'epsin hat Geselschap davon vor kurzer Zeit noch ein Beispiel mitgeteilt ${ }^{2}$ ).

Wie ich schon bemerkt habe, ist es nur Schein, dass die Labpräparate des Handels pepsinfrei sein sollten. Sobald die störenden Beimischungen entfernt sind, kommt die verdauende Kraft ans Licht. Andererseits scheint es, wie $\mathrm{Hammarsten}$ schon vor längerer Zeit gefunden hat, möglich, Pepsin wenigstens nahezu vollständig chymosinfrei zu machen, wenn nämlich die Lösung während einiger Zeit auf oder ein wenig uber Körpertemperatur erhitzt wird. Ich werde jetzt einige Beobachtungen mitteilen, aus welchen, wie ich glaube, hervorgeht, dass auch dieses nur Schein ist.

Auch vom Schwein herstammende Pepsinlösungen verlieren, wenn sie während einiger Zeit auf Körpertemperatur erwärmt werden, das Labungsvermögen. Eben deshalb überraschte es mich, dass aus 5 Tage bei Körpertemperatur mit $0,5 \% \mathrm{HCl}$ digerierter Magenschleimhaut bereitetes Pepsin kräftig labend wirkte, während die Digestionsflüssigkeit, aus welcher es sich ausgeschieden hatte, das labende Vermögen beinahe ganz verloren hatte.

Später zeigte Van $\mathrm{Dam}^{3}$ ), dass auch das gereinigte Schweinspepsin beim Erbitzen auf $42^{\circ} \mathrm{C}$. die labende Kraft beinahe völlig

1) Zeitschr. f. physiol. Chemie Bd. 94 S. 291.

2) Zeitschr. f. physiol. Chemie Bd. 94 S. 223.

3) Zeitschr. f. physiol. Chemie Bd. 64 S. 316. 
verliert, während die eiweissverdauende Kraft nur sehr wenig verringert wird. Dass aber auch hier von einer Trennung von Chymosin und Pepsin nicht die Rede sein konnte, stellte sich heraus, als er die digerierte Lösung halb mit Ammonsulfat sättigte und den dabei erhaltenen Niederschlag mittels Dialyse gegen $0,2 \% \mathrm{HCl}$ von Salz befreite. Die Enzymlösung hatte jetzt die labende Kraft zurückbekommen. Hieraus geht also hervor, dass bei der Digestion auf $42^{\circ} \mathrm{C}$. labungswidrige Stoffe gebildet werden, welehe mittels Halbsättigen mit Ammonsulfat nicht oder unvollständig zu fällen sind.

Ich habe untersucht, ob es sich auch in bezug auf das Infus aus Kalbsmagenschleimhaut nachweisen lässt, dass die in diesem Fall durch Frhitzung verursachte Trennung von Pepsin und Chymosin nur scheinbar ist. Zu dem Ende hahe ich versucht, das Infus, wenn die labende Kraft infolge von Erbitzung erheblich geschwächt war, von vielleicht gebildeten labungswidrigen Stoffen zu befreien. Mittels Dialyse gegen Wasser oder gegen Salzsäure gelang mir das nicht; ehensowenig erreichte ich meinen Zweck durch Fällung des Enzyms nittels Bleiessig und Ammon. Sättigung des Infuses mit Ammonsulfat hatte aber besseren Erfolg.

Ich verfuhr folgenderweise:

Die zerschnittene Magenschleimhaut eines neugehorenen Kalbes wurde in einem kühlen Zimmer nit 1 Liter $0,2 \%$ iger Salzsäure hingesetzt. Am folgenden Tage wurde ein kleiner Teil der Flüssigkeit abpipettiert und mit dem gleichen Volum $0,1 \% \mathrm{HCl}$ verdünnt. Von dieser Flüssigkeit wurde erstens die labende Kraft bestimmt durch Mischung von $1 \mathrm{cem}$ mit $10 \mathrm{ccm}$ zuror auf $38^{\circ} \mathrm{C}$. gebrachter Mileh. Zweitens wurde nach der Grützner'schen Methode in der von Geselschap beschriebenen Weise ${ }^{1}$ ) die verdauende Kraft bestimmt. Immer wurden $50 \mathrm{mg}$ Karminfibrinpulver mit $9 \mathrm{cem} 0,1 \%$ iger HCl nach wenigstens 10 Minuten Stehen, damit das Fibrin die Zeit hatte, vollständig zu schwellen, mit $1 \mathrm{ccm}$ der zu prüfenden Lösung gemischt. Jede Minute wurde das mit dem Daumen geschlossene Röhrchen einmal umgedreht, um das Fibrin gleichmässig mit dem lepsin in Berührung zu bringen. Nach 10 bis 15 Minuten, je nachdem die Temperatur der Umgebung höher oder niedriger war, wurde die Verdaung mittels Filtration dureh Glaswolle unterbrochen. Zu gleicher Zeit wurden 50 mg Karminfibrin ganz in derselben Weise

1) Zaitschr. f. physiol. Chemie Bd. 94 S. 207. 
mit $1 \mathrm{ccm}$ einer Schweinspepsinlösung in $0,1 \% \mathrm{HCl}$, welche auf $1 \mathrm{ccm} 0,1 \mathrm{mg}$ Pepsin enthielt, verdaut. $0,5 \mathrm{mg}$ dieses Pepsins in $10 \mathrm{ccm} 0,2 \%$ iger $\mathrm{HCl}$ verdaute, nach der Mett'schen Methode untersucht, in 24 Stunden $5,5 \mathrm{~mm}$. Nach dem Filtrieren durch Glaswolle wurden beide Lösungen mit einer verdauten Karminlösung willkürlicher Konzentration im Grützner'schen Kolorimeter verglichen. Auf diese Weise konnte der Pepsingehalt in einem bestimmten Maasse ausgedrückt werden. Nach je 4 oder 5 Tagen wurde eine neue $0,1 \%$ ige Pepsinlösung in $0,2 \% \mathrm{HCl}$ gemacht. Vor dem Gebrauch wurde $1 \mathrm{ccm}$ hiervon mit $9 \mathrm{cem} 0,1 \%$ igem $\mathrm{HCl}$ verdünnt. Das Pepsin wurde im Exsikkator im Dunkeln aufbewahrt. Ich konnte mich deshalb darauf verlassen, dass der Pepsingehalt immer in demselben Maasse ausgedrückt wurde.

Nachdem die Magenschleimhaut mit Salzsäure einen Tag an einem kühlen Ort gestanden hatte, wurde die Flüssigkeit, bisweilen nach Kolieren, meistens mit der Schleimhaut, auf $38^{\circ} \mathrm{C}$. gebracht. War die Sehleimhaut mit der Flüssigkeit in Berührung gelassen, so war sie bald ganz verdaut. Täglich, oder jeden zweiten Tag, wurde die labende und die verdauende Kraft bestimmt. Immer sank die erstere viel schneller und mehr als die zweite, wenn auch das Herabsinken im einen Fall viel beträchtlicher war als im andern. Nach einigen Tagen wurde die Flüssigkeit durch zusammengepressten Filtrierpapierbrei abgesogen. Dieses Filtrat wurde, bisweilen nach Dialyse gegen destilliertes Wasser, mit Ammonsulfat gesättigt. Wenn auch gereinigtes Pepsin dureh Halbsättigen mit Ammonsulfat vollständig gefällt wird, gelingt dies, wie ich früher mitgeteilt babe, nicht, wenn die Lösung viel Albumosen enthält. Aus dem Kalbsmageninfus konnte ich sogar, auch nach vollständiger Sättigung mit Ammonsulfat, nicht immer ein klares Filtrat erhalten. Der Niederschlag, welcher jetzt in $0,1 \% \mathrm{HCl}$ gelöst und gegen Salzsäure derselben Konzentration dialysiert wurde, enthielt also keineswegs alles Enzym, welches in der Verdauungslösung enthalten war. Aus dem Verhältnis der labenden und der verdauenden Kraft konnte aber beurteilt werden, ob die Chymosinwirkung tatsächlich so stark verringert war, wie es aus der Prüfung des erwärmten Infuses hervorzugehen schien.

Ich lasse hier einige Ergebnisse folgen:

Frisch bereitetes Infus. $10 \mathrm{ccm}$ mit $10 \mathrm{ccm} 0,1 \%$ igem $\mathrm{HCl}$. Hiervon: $1 \mathrm{ccm}$ mit $10 \mathrm{ccm}$ Milch; nach einigen Sekunden geronnen. 
1 .cm mit $50 \mathrm{mg}$ Karminfibrin und $9 \mathrm{ccm} 0,1 \%$ iger $\mathrm{HCl}$; Kolorimeter 6,0 . $1 \mathrm{ccm} 0,1 \%$ iges Pepsin mit $50 \mathrm{mg}$ Karminfibrin and $9 \mathrm{ccm} 0,1 \%$ iger $\mathrm{HCl}$; Kolorimeter 5,7 .

Das verdünnte Infus besitzt also die verdauende Kraft einer Lösung von etwas mehr als $0,1 \mathrm{mg}$ Pepsin per Kubikzentimeter. Das Infus auf $38^{\circ} \mathrm{C}$. erwärmt, nach 6 Tagen filtriert. $460 \mathrm{ccm}$ des klaren Filtrates 24 Stunden gegen destilliertes Wasser dialysiert. Hiervon $10 \mathrm{ccm}$ mit $10 \mathrm{ecm} 0,1 \%$ iger $\mathrm{HCl}$ verdünnt.' $1 \mathrm{ccm}$ mit $10 \mathrm{ccm}$ Milch; nach 3 Standen nicht geronnen. $1 \mathrm{ccm}$ mit $50 \mathrm{mg}$ Karminfibrin und

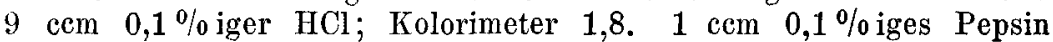
mit $50 \mathrm{mg}$ Karminfibrin und $9 \mathrm{ccm} 0,1 \%$ iger $\mathrm{HCl}$; Kolorimeter 3,3 . Pepsin.

Das dialysierte Infus enthielt also per Kubikzentimeter $0,055 \mathrm{mg}$

$450 \mathrm{ccm}$ mit Ammonsulfat gesättigt. Der Niederschlag wurde in $0,1 \% \mathrm{HCl}$ gelöst und gegen Salzsäure derselben Konzentration dialysiert. Die filtrierte, salzarme Iösung wurde mit $0,1 \% \mathrm{HCl}$ so weit verdünnt, dass $1 \mathrm{ccm}$ wieder eine verdauende Kraft $0,055 \mathrm{mg}$ Pepsin entsprechend, zeigte.

Jetzt verursachte $1 \mathrm{ccm}$ mit $10 \mathrm{ccm}$ Milch vollständige Gerinnung in 21 Minuten.

Infus 10 Tagen hei $38^{\circ} \mathrm{C}$. digeriert, dann tittriert und gegen destilliertes Wasser dialysiert. 1 cem der mit dem gleichen Volum $1 \%$ igcr $\mathrm{HCl}$ verdünnten Flüssigkeit legt $10 \mathrm{ccm}$ Milch in 40 Minuten fest. Pepsingehalt $0,081 \mathrm{mg}$.

$475 \mathrm{ccm}$ der dialysierten Flüssigkeit mit Ammonsulfat gesättigt. Der Niederschlag (viel Verlust beim Filtrieren) in gewohnter Weise in 0,1\% HCl aufgenommen und dialysiert. Volum $220 \mathrm{ccm}$. Während $1 \mathrm{ccm}$ dieser mit $1 \mathrm{Vol}$. 0,1\% iger HCl verdünnter Lösnng eine vordauende Kraft zeigte, nur $0,048 \mathrm{mg}$ Pepsin jer Kubikzentimeter entsprechend, machte $1 \mathrm{ccm} 10 \mathrm{ccm} \mathrm{Milch}_{\perp}$ in 75 Sekunden gerinuen.

Frisch horeitetos Infus. Gerinnungszeit und Verdanungsvermögen in der üblichcn Weise bestimmt resp. 15 Sckunden und $0,12 \mathrm{mg}$ Pepsin. Nach zwölftägiger Lrwärmung auf $38^{\circ} \mathrm{C}$., $71 / 2$ Minute und $0,093 \mathrm{mg}$ Pepsin. Die Flüssigkeit 24 Stunden auf $45^{\circ} \mathrm{C}$. erwärmt. Jetzt war die Gerinnungszeit $1^{1}$; stunde und der Pepsingehalt $0,066 \mathrm{mg}$.

Ohne vorhergehende Dialyse wurde das Infus mit Ammonsulfat gesättigt. Der Niederschlag, wovon viel verloren ging, aufgelöst und von Salz befreit, zcigte eine verdauende Kraft, welche nur $0,05 \mathrm{mg}$ Pepsin entsprach. War also bei dor Erhitzung wirklich so viel Chymosin zerstört worden, als es bei der Prüfung des Infuses den Anschein hatte, so müsste die Gerinnungszeit beträchtlich länger als $1^{1 / 2}$ Stunde erwartet werden. Die Milch war aler in 52 Minuten vollständig geronnen.

Infus 5 Tage bei $38^{0} \mathrm{C}$. digeriert. Gerinnungszeit 30 Minuten. l'epsingehalt per Kubikzentimeter $0,1 \mathrm{mg}$.

Infus filtriert und $200 \mathrm{ccm}$ mit Ammonsulfat gesättigt. Der Niclerschlag in $0,1 \%$ iger Salzsäurie gểöst und von Salz befreit. Gerinnungszeit 5 Minuten einem Pepsingehalt von $0,107 \mathrm{mg}$ entsprechend. 
$760 \mathrm{ccm}$ eines 9 Tage auf $38^{\circ} \mathrm{C}$. erwärmten, dann filtrierten, gegen destilliertes Wasser dialysierten und nochmals filtrierten Infuses, welches erst nach $3^{3} / 4$ Stunden Gerinnung hervorruft und eine verdauende Kraft $0,064 \mathrm{mg}$ Pepsin per Kubikzentimeter entsprechend besitzt, wird mit Bleiessig und Ammoniak behandelt. Der Niederschlag wird mittels Oxalsäure zersetzt und die abfiltrierte Flüssigkeit gngen strömendes Leitungswasser bis zur schwach-sauren Reaktion dialysiert. Das Volum der dialysierten und filtrierten Lösung beträgt $37 \mathrm{ccm}$. $5 \mathrm{ccm}$ derselben werden mit $5 \mathrm{ccm} 0,1 \%$ iger $\mathrm{HCl}$ verdünnt. Darin entspricht der Pepsingehalt $0,147 \mathrm{mg}$ per Kubikzentimeter, während $1 \mathrm{ccm}$ mit $10 \mathrm{ccm}$ Milch nach 12 Minuten einen Anfang von Gerinnung hervorruft, welche erst nach 18 Minuten vollständig ist. Die labende Kraft ist also der verdauenden gegenüber sehr gering. $25 \mathrm{ccm}$ der Lösung werden mit Ammonsulfat gesättigt. Die in der üblichen Weise behandelte Lösung des Niederschlages wird mit $0,1 \% \mathrm{HCl}$ bis zu $25 \mathrm{ccm}$ angefüllt. Von der so erhaltenen Lösung werden $5 \mathrm{ccm}$ mit $5 \mathrm{ccm} 0,1 \%$ iger $\mathrm{HCl}$ verdünnt. Jetzt ist die Gerinnungszeit 340 Sekunden während die verdauende Kraft $0,124 \mathrm{mg}$ Pepsin per Kubikzentimeter entspricht.

Aus diesen Befunden muss, wie es mir scheint, gefolgert werden. dass bei der Erwärmung auf Körpertemperatur nicht Chymosin, im Gegensatz zu Pepsin, zerstört wird, sondern dass die Einwirkung auf Kasein hindernde Stoffe gebildet werden, Stoffe, welche von Bleiessig und Ammoniak mit dem Enzym gefällt, mittels Dialyse gegen Wasser oder Salzsäure nicht oder nur sehr unvollständig entfernt werden, beim Fällen des Enzyms mit Ammonsulfat aber zum grössten Teil gelöst bleiben. Man würde anders genötigt sein anzunehmen, dass bei der Behandlung mit Ammonsulfat neues Chymosin gebildet würde. Obgleich, wie aus den Pepsinbestimmungen hervorgeht, das eine Mal viel, das andere weniger Enzym verloren geht, ist ja immer die Einwirkung des mittels Ammonsulfat gereinigten Enzyms auf Milch kräftiger als vor der Reinigung, wenn auch die Reinigung des Enzyms aus Kalbsmagenschleimbaut immer noch viel zu wünschen übrig lässt. Bei der Annahme, dass die Gerinnung der Milch demselhen Enzym, Pepsin, zuzuschreiben ist, das auch Eiweis zu verdauen imstande ist, sind die hier mitgeteilten Beobachtungen erklärlich. Man braucht dabei nur in Betracht zu ziehen, dass die beiden Wirkungen unter sehr verschiedenen Verhältnissen stattfinden. Bei der Gerinnung wird, bei neutraler oder sehr schwach-saurer Reaktion, aus einem ganz eigentümlichen Eiweisskörper, Kasein, Parakasein gebildet; bei der Verdauung dagegen werden, bei einem viel grösseren Überschuss von H-Ionen, Eiweissstoffe sehr verschiedener Art in Albumosen gespalten. Man braucht sich darüber nicht zu 
Über die Frage, ob neben dem Pepsin ein anderes Enzym usw.

wundern, dass die Wirkung des Enzyms in einem Fall von Beimischungen gehemmt wird, welche im anderen unschädlich sind.

Bei der Annahme von zwei unter sich verschiedenen Enzymen scheint es mir nicht recht begreiflich, wie in den verschiedenen Versuchen eine Vermehrung der Chymosinmenge zustande gekommen sein kann.

Die Zuverlässigkeit der Bestimmungen der labenden und der verdauenden Kraft scheint mir für die aus den Versuchen gezogenen Folgerungen genügend. Zwar wurde jeden Tag andere Milch für die Gerinnungsprobe gebraucht. Daraus können aber nur kleine Unterschiede hervorgehen. Bei der nahezu täglichen Prüfung der erwärmten Infuse wurden nur ejnzelne Male Störungen in der Regelmässigkeit der Verringerung der labendeu Kraft beobachtet. Zudem waren diese Störungen sehr geringfügig im Vergleich mit den oben erwähnten grossen Unterschieden. Durch Verdünnen der zu prüfenden sauren Lösungen mit dem gleichen Volum $0,1 \% \mathrm{HCl}$ wurde dafür Sorge getragen, dass beim Vermischen von $1 \mathrm{ccm}$ mit $10 \mathrm{~cm}$ Milch einigermaassen in Betracht kommenden Schwankungen im Gehalt an $\mathrm{H}$-Ionen nicht vorkamen. Die Milch und auch die Enzymlösung wurden immer auf $38^{\circ} \mathrm{C}$. vorgewärmt und sogleich nach dem Zusatz gut gemischt.

Für die Pepsinbestimmung genügt die $\mathrm{G} r$ üt $\mathrm{z}$ ner'sche Methode sicher den Anforderungen, welche in hezug auf Genauigkeit bei Versuchen, wie die hier hesehriebenen, zu stellen sind. Auch hierbei kamen unerwartete $A b$ weichungen sehr selten vor und dann noch in unbedeutendem Maasse. Ofters habe ich die Resultate durch Anwendung der Mett'schen Methode kontrolliert und immer sehr befriedigende Übereinstimmung gefunden. Das als Standard gebrauchte Pepsin wurde inmer demselben Vorrat entnonmen und genau ehenso lange Zeit und in derselben Weise mit demselben Karminfibrin in Berührung gelassen.

Die Frage, ob man das Recht hat, Pepsin und Chymosin als wwei verschiedenartige Enzyme zu betrachten, ist selbstverständlich mit dem oben Gesagten nicht entschieden. Die sehr interessanten Beobachtungen Hammarsten's sind wohl, von dem unitarischen Standpunkt betrachtet, augenblicklich nicht in befriedigender Weise zu erklären. Dennoch darf es nicht unberücksichtigt bleiben, dass das von Hammarsten aus der Magenschleimhaut des Kalbes bereitete Enzym sicher nicht so gut gereinigt war als das beim Pepsiu 
des Hundes und des Schweines möglich ist, welches sich im Dialysator in durchsichtigen, anscheinend homogenen Kügelchen absetzt, während das Enzym des Kalbes sich in klebrigen Flocken ausscheidet.

H a mmarsten fand, dass ein von Alkali geschädigtes Enzym, welches Fibrin so gut wie nicht verdaute, Milch aber in kurzer Zeit festlegte, in einer $0,03 \%$ igen Lösung von $\mathrm{HCl}$ Kasein viel kräftiger in Albumosen spaltete als bei neutraler Reaktion und schliesst daraus, dass das Enzym keine Verunreinigungen enthielt, welche bei saurer Reaktion die proteolytische Wirkung hinderten. Darf man aber die Wirkung des Enzyms auf Kasein mit derjenigen, bei viel stärker sauren Reaktion, auf Fibrin oder Hühnereiweiss gleich setzen? Ich glaube, dass die Untersuchungen von $\mathrm{Van} \mathrm{Dam}^{1}$ ) zu einer verneinenden Antwort auf diese Frage Veranlassung geben.

Es scheint mir kaum zu bestreiten zu sein, dass Pepsin und auch andere proteolytische Enzyme bei neutraler oder schwachsaurer Reaktion aus Kasein Parakasein bilden können. In mehr als einer Hinsicht, auch in den hier beschriebenen Versuchen, hat es sich herausgestellt, dass man aus abweichenden Erfahrungen beim Enzym des Kalbes unrichtigerweise geschlossen hat, dass dieselbe Wirkung auch noch von einem andersartigen Enzym verursacht werden kann. Die Vermutung, dass jetzt noch nicht erklärte Abweichungen in bezug auf dieses Enzym Verunreinigungen 7.ugeschrieben werden müssen, ist, meiner Ansicht nach, bei dem gegenwärtigen Stand der Kenntnis nicht von der Hand zu weisen.

1) Zeitschr. f. physiol. Chemie Bd. 61 s. 147 , Bd. 64 S. 316, Bd. 79 S. 247. 